\title{
Challenges in the management of neuroendocrine cervical cancer during pregnancy: A case report
}

\author{
BLANCA GIL-IBAÑEZ ${ }^{1}$, PURIFICACION REGUEIRO ${ }^{2}$, ELISA LLURBA ${ }^{3}$, \\ LORENA FARIÑAS-MADRID ${ }^{4}$, ANGEL GARCIA ${ }^{5}$ and BERTA DIAZ-FEIJOO ${ }^{6}$
}

\begin{abstract}
${ }^{1}$ Unit of Gynecological Oncology, Institute Clinic of Gynecology, Obstetrics and Neonatology (ICGON), Hospital Clinic of Barcelona, 08036 Barcelona; ${ }^{2}$ Department of Obstetrics and Gynecology, General Hospital of Hospitalet, 08906 Barcelona; ${ }^{3}$ Department of Obstetrics and Gynecology, Sant Pau University Hospital, Autonomous University of Barcelona, 08041 Barcelona; ${ }^{4}$ Department of Oncology, Vall d'Hebron Institute of Oncology (VHIO); ${ }^{5}$ Department of Pathology,

Vall d'Hebron University Hospital; ${ }^{6}$ Unit of Gynecological Oncology, Department of Obstetrics and Gynecology,

Hospital Materno-Infantil Vall d'Hebron, Autonomous University of Barcelona, 08035 Barcelona, Spain
\end{abstract}

Received June 25, 2018; Accepted September 11, 2018

DOI: $10.3892 / \mathrm{mco} .2018 .1717$

\begin{abstract}
Large-cell neuroendocrine carcinoma (LCNEC) is an uncommon histological subtype of cervical cancer that is associated with poor survival and its occurrence during pregnancy is particularly rare. We herein present the case of a female patient who was diagnosed with cervical LCNEC during pregnancy. The patient declined pregnancy termination and was treated with neoadjuvant chemotherapy with cisplatin and etoposide, without associated toxicity and with good fetal development. At 31.4 weeks of gestation, the fetus was delivered by caesarean section, and the patient underwent radical nerve-sparing hysterectomy with bilateral adnexectomy, along with pelvic and inframesenteric para-aortic lymphadenectomy. The patient received adjuvant chemoradiotherapy and there was no evidence of recurrence or metastasis at 38 months postoperatively. The baby has also been followed up, without any signs of neurodevelopmental disorders. To the best of our knowledge, the present report describes the first case of LCNEC with pregnancy-preserving management in the literature to date.
\end{abstract}

\section{Introduction}

The incidence of cancer diagnosis during pregnancy is estimated to be 1 in every 1,000 pregnant women. Breast cancer, melanoma

Correspondence to: Dr Blanca Gil-Ibáñez, Unit of Gynecological Oncology, Institute Clinic of Gynecology, Obstetrics and Neonatology (ICGON), Hospital Clinic of Barcelona, Villarroel 170, 08036 Barcelona, Spain

E-mail: blgil@clinic.cat

Key words: uterine cervix, cervical cancer in pregnancy, neuroendocrine carcinoma, radical hysterectomy post-caesarean section, neoadjuvant chemotherapy and cervical cancer are the most commonly diagnosed types of cancer during pregnancy (1). Large-cell neuroendocrine carcinoma (LCNEC) is an uncommon histological subtype (0.5-3\%) of cervical cancer associated with poor survival, whereas the occurrence of LCNEC during pregnancy is particularly rare. Although recent data support the use of perioperative chemotherapy, in particular platinum, with or without etoposide, to improve the survival of patients with LCNEC (2), treatment during pregnancy remains completely experimental. To the best of our knowledge, the present report describes the first case of LCNEC in the literature to date that was successfully managed with a pregnancy-preserving approach.

\section{Case report}

A 34-year-old woman, gravida 3, para 3, was referred to Hospital Clinic de Barcelona (Barcelona, Spain) in March, 2015 with a biopsy result of NEC of the cervix at 21 weeks of gestation. The patient had no history of medical conditions or previous surgery. Gynecological examination revealed a 3-cm exophytic cervical tumor, without parametrial invasion, but with involvement of the anterior vaginal fornix. Legal interruption of the pregnancy was offered, but the patient declined due to her religious beliefs.

Ultrasonographic examination revealed a cervical tumor sized 40x19x35 mm, with involvement of the anterior fornix of the vagina, without evidence of parametrial invasion, and a normally growing fetus with normal hemodynamic parameters. Magnetic resonance imaging (MRI) revealed no evidence of lymph node involvement or metastatic disease (Fig. 1). The tumor was classified as clinical stage IIA1 according to the guidelines of the International Federation of Gynecology and Obstetrics.

On histological examination of biopsy material, the mass was diagnosed as high-grade cervical LCNEC, and immunohistochemistry revealed positive staining for chromogranin, synaptophysin, CD56, cytokeratin (CK) 7 and K-i67 (>80\%), and negative for $\mathrm{p} 63$. The levels of carcinoembryonic antigen 
and squamous cell carcinoma antigen were within the normal range. Human papillomavirus (HPV) 18 was identified by polymerase chain reaction analysis.

The patient received neoadjuvant chemotherapy (NACT) with 3 cycles of cisplatin (CDDP; $50 \mathrm{mg} / \mathrm{m}^{2}$ ) and etoposide (VP-16; $100 \mathrm{mg} / \mathrm{m}^{2}$ ) every 3 weeks, without associated toxicity and with good fetal development. The patient was clinically followed every 2 weeks with ultrasonographic and gynecological examinations. Fetal control was regularly performed with ultrasound and Doppler examination. The tumor remained stable during that period.

At 31.4 weeks, the patient underwent radical hysterectomy with nerve sparing (type $\mathrm{C} 1$ ), along with bilateral adnexectomy and pelvic and inframesenteric para-aortic lymphadenectomy (Fig. 2). The fetus was delivered with caesarian section, without complications or morphological malformations. There were no intraoperative or postoperative complications. Within the first 2 days the patient had post-void residual urine volume $<100 \mathrm{ml}$. The hospital stay was 6 days.

The surgical specimen displayed a high-grade cervical LCNEC sized 40x16 mm, with positive lymphovascular space invasion, but without parametrial, vaginal, placental or lymph node involvement (pelvic nodes $0 / 22$, para-aortic nodes $0 / 18$ ). The surgical margins were negative (Fig. 3).

The patient completed treatment with chemoradiation (intensity-modulated radiotherapy at $45 \mathrm{~Gy} / 15 \mathrm{fr}$ and 5 cycles of concomitant weekly CDDP at $40 \mathrm{mg} / \mathrm{m}^{2}$ ); she was unable to receive the sixth cycle due to the development of G3 asthenia after the fifth cycle.

During follow-up, physical examination, cytology, measurement of tumor marker levels and computed tomographic examination revealed no evidence of recurrence or metastasis at 38 months postoperatively. The baby has also been followed up, without any signs of neurodevelopmental disorders in July 2018.

\section{Discussion}

Over the past decades, there has been a rising trend of pregnancy at more advanced ages; therefore, cancer during gestation is an increasing problem in clinical practice. Cervical cancer is the second most commonly diagnosed cancer during pregnancy, following breast cancer. NEC of the cervix appears to occur in women who are younger compared with those with the more common HPV-associated cervical cancers; therefore, pregnancy may be a more serious concern for such patients (3).

A growing body of evidence indicates that gestation does not adversely affect the prognosis of cervical cancer. In 2009, Stensheim et al (4), using data from the Cancer Registry and the Medical Birth Registry of Norway, investigated whether cervical cancer diagnosed during pregnancy or lactation was associated with increased risk of cause-specific mortality. No increase in the risk of cause-specific mortality (hazard ratio $=0.89$; 95\% confidence interval: $0.52-1.53$ ) in patients diagnosed during pregnancy was observed in the multivariate analyses adjusted for age at diagnosis, extent of disease and diagnostic period, with a median follow-up of 10.8 years (4). Pettersson et al (5) in 2010, using the records at the Radiumhemmet (Solna, Sweden) between 1914 and 2004, compared the survival of 41 patients diagnosed with cervical cancer while pregnant or within 6 months postpartum with 82 non-pregnant women matched for age, stage and histopathology. No significant difference in 10-year survival rates was observed between the two groups.

Based on these findings, and taking into consideration the patient's wishes and our extensive experience with treating breast cancer diagnosed during pregnancy, we decided to proceed with treatment without pregnancy interruption. A multidisciplinary approach between Obstetrics and Gynecological Oncology groups in such cases is crucial for optimizing maternal treatment and fetal protection (6-8).

The European Society of Medical Oncology and European Society of Gynecological Oncology (ESGO) have published guidelines on gynecological cancer in pregnancy, in which treatment of cervical cancer during gestation is indicated, albeit experimental. It is important to note that patients who wish to preserve their pregnancy should be informed that the treatment will be individualized out of the standard process. In these guidelines, when cervical cancer stage is higher than IB2 and the nodes are negative, NACT is considered as the only method for preserving pregnancy until fetal maturity.

The therapeutic value of pelvic staging lymphadenectomy prior to the initiation of NACT remains unknown. The ESGO guidelines indicate that it may be safely performed between the 13th and 22nd weeks of gestation to assess pelvic nodal status $(1,9)$. However, it was decided against in the present case, as MRI during pregnancy has also demonstrated a good positive predictive value for nodal metastasis (10).

The Society of Gynecological Oncology guidelines published in 2011 recommend multimodal management for all stages of NEC of the cervix (11), and the majority of patients receive some combination of surgery, radiation and chemotherapy. For women with bulky lesions, NACT followed by radical hysterectomy with lymphadenectomy plus adjuvant chemotherapy \pm radiotherapy is a viable option (12).

Exposure to chemotherapy during the first trimester of pregnancy increased the risk of fetal defects compared with administration during the second and third trimesters, which has not been associated with significant adverse effects in the fetus in the short or long term; however, not all chemotherapeutic agents are safe (1).

No standard treatment for LCNEC has yet been established (13), and the regimen with CDDP and VP-16 was selected based on the management of small-cell lung cancer $(2,14)$.

In the NTP Monograph on Cancer Chemotherapy during pregnancy, published by the U.S. Department of Health and Human Services in May 2013, CDDP is a widely accepted treatment during pregnancy at doses ranging from $20 \mathrm{mg} / \mathrm{m}^{2}$ for 5 days up to $75 \mathrm{mg} / \mathrm{m}^{2}$ every 3 weeks (15). The rate of malformations following exposure to CDDP in the first trimester was $20 \%$; however, in the second or third trimester of pregnancy, that percentage decreased to $1 \%$. The most frequent minor malformations were blepharophimosis, microcephaly and hydrocephalus. CDDP exposure is associated with decreased intrauterine growth by $13 \%$. Data on the use of VP-16 during pregnancy are limited: A 3\% rate of major malformations following administration in the second and third trimesters and $24 \%$ fetal growth restriction have been reported. The most frequent major malformation was cerebral atrophy and ventriculomegaly $(9,16)$. 


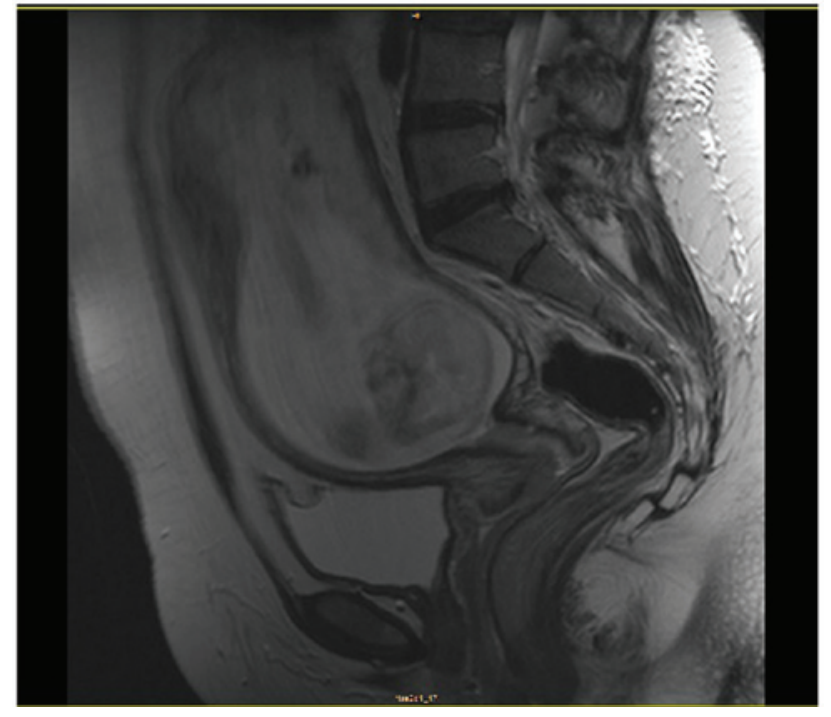

Figure 1. On magnetic resonance imaging examination there was no evidence of lymph node involvement or metastatic disease.
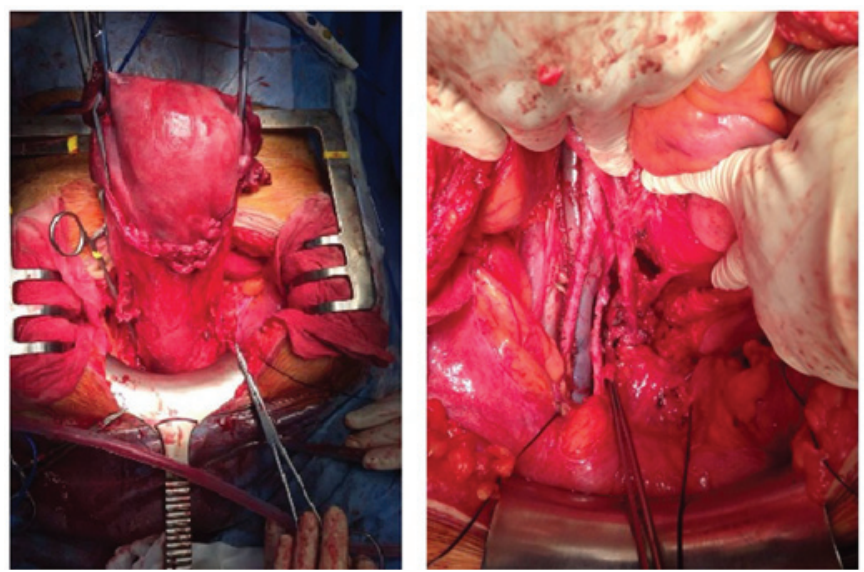

Figure 2. Intraoperative images during caesarean section performed at 31.4 weeks, with concomitant radical nerve-sparing hysterectomy, bilateral adnexectomy and pelvic and inframesenteric para-aortic lymphadenectomy.

In the present case, based on the advice of the multidisciplinary team, the patient received three cycles of NACT with CDDP $50 \mathrm{mg} / \mathrm{m}^{2}$ and VP-16 $100 \mathrm{mg} / \mathrm{m}^{2}$ every 3 weeks.

Due to the aggressive nature of cervical NECs, surveillance with tumor assessment was performed every 2 weeks, including physical examination and vaginal ultrasound, always performed by the same Gynecological Oncology specialist sonographer. No more MRI scans were indicated, as the tumor was stable. Fetal well-being was evaluated by the High Pregnancy Risk Unit, with fetal growth assessment every 2 weeks. During surveillance, NACT appeared to stabilize the tumor, preventing dissemination without harming the fetus.

Although it remains unclear when is the best time to deliver the fetus, the general trend is after 28 weeks of gestation or after 3 cycles of chemotherapy. Regardless, a 3-week period should be allowed between the last chemotherapy dose and the expected date of delivery, 33.2 weeks on average. We decided to deliver after the third cycle of NACT,

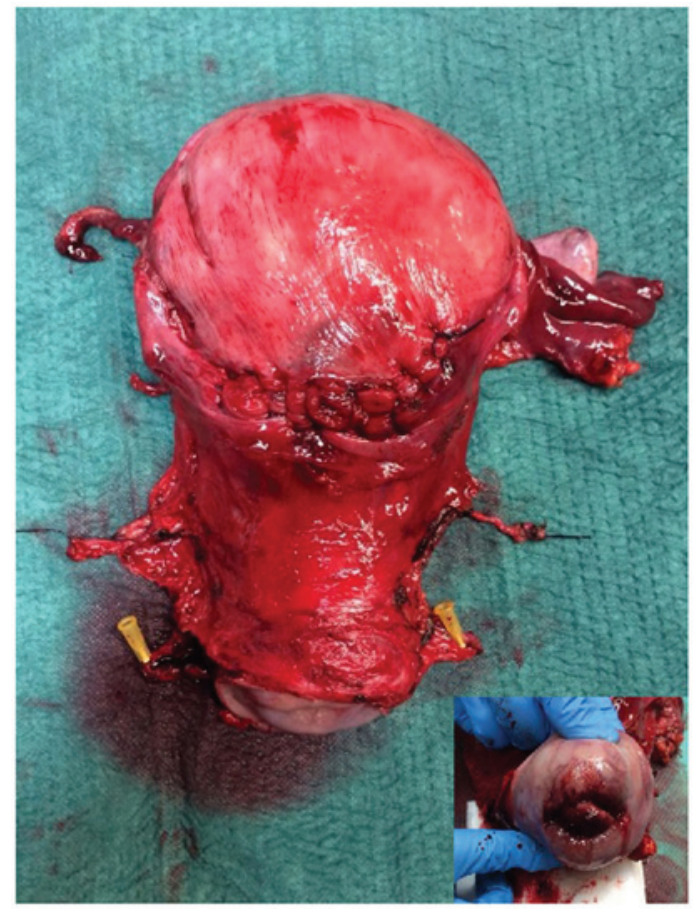

Figure 3. The surgical specimen displayed a high-grade cervical large-cell neuroendocrine carcinoma, sized 40×16 mm (inset).

at 31 weeks of gestation, and radical surgery was performed concomitantly with caesarean section (1). Nerve-sparing radical hysterectomy type $\mathrm{C} 1$ with complete tumor resection was performed, in order to decrease the risk of bladder, rectal and sexual dysfunction. The identification and preservation of hypogastric and pelvic splanchnic nerves and the pelvic plexus after caesarean section was feasible without increasing the volume of blood loss.

In a recent study by Stecklein et al investigating survival in cervical NEC, $80 \%$ of the patients progressed and $70 \%$ succumbed to the disease during a median follow-up of 21.5 months [5-year progression free survival (PFS) rate of 20\%, and 5-year overall survival (OS) rate of 27\%] (17). The patient in the present case remained disease-free after 32 months of follow-up. In that same study, patients with cervical LCNEC had significantly better median PFS (median not reached vs. 10.0 months, $\mathrm{P}=0.02$ ) and exhibited a trend toward better median OS (153 vs. 21 months, $\mathrm{P}=0.08$ ) compared with patients with other histological types (17).

It may be concluded that NACT appears to be a viable option for preserving pregnancy in advanced cases, while stabilizing the disease and preventing tumor dissemination before reaching fetal maturity (sufficient gestational age and stage of fetal development). The present case demonstrated that NACT followed by radical hysterectomy concomitantly with caesarean section may be considered as a pregnancy-sparing treatment option. In several countries with specialized multidisciplinary teams, this may be a feasible alternative to pregnancy termination.

\section{Acknowledgements}

Not applicable. 


\section{Funding}

No funding was received.

\section{Availability of data and materials}

Not applicable.

\section{Authors' contributions}

BGI reviewed the literature, conducted data collection, surgery and prepared the manuscript. PR reviewed the literature, conducted data collection. ELL reviewed the literature, performed fetal monitoring during pregnancy. LFM reviewed the literature, conducted medical oncology analysis. AG performed pathological examinations and critically revised the manuscript for intellectual important content. BDF reviewed the literature, conducted data collection, and surgery, and critically revised the manuscript for intellectual important content. All authors approval of the final version of the manuscript.

\section{Ethics approval and consent to participate}

Not applicable.

\section{Patient consent for publication}

The patient agreed with the publication of the case and has named her daughter Milagros (miracle).

\section{Competing interests}

The authors declare that they have no competing interests to disclose.

\section{References}

1. Peccatori FA, Azim HA Jr, Orecchia R, Hoekstra HJ, Pavlidis N, Kesic V and Pentheroudakis G; ESMO Guidelines Working Group: Cancer, pregnancy and fertility: ESMO Clinical Practice Guidelines for diagnosis, treatment and follow-up. Ann Oncol 24 (Suppl 6): vi160-vi170, 2013.

2. Embry JR, Kelly MG, Post MD and Spillman MA: Large cell neuroendocrine carcinoma of the cervix: Prognostic factors and survival advantage with platinum chemotherapy. Gynecol Oncol 120: 444-448, 2011.

3. Frumovitz M: Small- and large-cell neuroendocrine cervical cancer. Oncology (Williston Park) 30: 70, 77-78, 93, 2016.
4. Stensheim H, Møller B, van Dijk T and Fosså SD: Cause-specific survival for women diagnosed with cancer during pregnancy or lactation: A registry-based cohort study. J Clin Oncol 27: 45-51, 2009.

5. Pettersson BF, Andersson S, Hellman K and Hellström AC: Invasive carcinoma of the uterine cervix associated with pregnancy: 90 years of experience. Cancer 116: 2343-2349, 2010.

6. Córdoba O, Llurba E, Cortés J, Sabadell MD, Lirola JL, Ferrer Q and Xercavins J: Complete pathological remission in a patient with hormone-receptor positive and c-erbB-2 expression-negative breast cancer treated with FAC chemotherapy during pregnancy. Tumori 96: 629-632, 2010.

7. Córdoba O, Bellet M, Vidal X, Cortés J, Llurba E, Rubio IT and Xercavins J: Pregnancy after treatment of breast cancer in young women does not adversely affect the prognosis. Breast 21 : 272-275, 2012.

8. Córdoba O, Llurba E, Saura C, Rubio I, Ferrer Q, Cortés J and Xercavins J: Multidisciplinary approach to breast cancer diagnosed during pregnancy: Maternal and neonatal outcomes. Breast 22: 515-519, 2013.

9. Amant F, Halaska MJ, Fumagalli M, Dahl Steffensen K, Lok C, Van Calsteren K, Han SN, Mir O, Fruscio R, Uzan C, et al: Gynecologic cancers in pregnancy: Guidelines of a second international consensus meeting. Int J Gynecol Cancer 24: 394-403, 2014.

10. Balleyguier C, Fournet C, Ben Hassen W, Zareski E, Morice P, Haie-Meder C, Uzan C, Gouy S, Duvillard P and Lhommé C: Management of cervical cancer detected during pregnancy: Role of magnetic resonance imaging. Clin Imaging 37: 70-76, 2013.

11. Gardner GJ, Reidy-Lagunes D and Gehrig PA: Neuroendocrine tumors of the gynecologic tract: A Society of Gynecologic Oncology (SGO) clinical document. Gynecol Oncol 122: 190-198, 2011.

12. Plante M: Bulky early-stage cervical cancer ( $2-4 \mathrm{~cm}$ lesions): Upfront radical trachelectomy or neoadjuvant chemotherapy followed by fertility-preserving surgery: Which is the best option? Int J Gynecol Cancer 25: 722-728, 2015.

13. Delaloge S, Pautier P, Kerbrat P, Castaigne D, Haie-Meder C, Duvillard P, Guivarch C, Goupil A, Borel C and Lhommé C: Neuroendocrine small cell carcinoma of the uterine cervix: What disease? What treatment? Report of ten cases and a review of the literature. Clin Oncol (R Coll Radiol) 12: 357-362, 2000.

14. Zivanovic O, Leitao MM Jr, Park KJ, Zhao H, Diaz JP, Konner J, Alektiar K, Chi DS, Abu-Rustum NR and Aghajanian C: Small cell neuroendocrine carcinoma of the cervix: Analysis of outcome, recurrence pattern and the impact of platinum-based combination chemotherapy. Gynecol Oncol 112: 590-593, 2009.

15. National Toxicology Program: NTP Monograph: Developmental Effects and Pregnancy Outcomes Associated With Cancer Chemotherapy Use During Pregnancy. https://ntp.niehs.nih.gov/ pubhealth/hat/noms/chemo/index.html. Accessed May 13, 2013.

16. Correa A, Cragan JD, Kucik JE, Alverson CJ, Gilboa SM, Balakrishnan R, Strickland MJ, Duke CW, O'Leary LA, Riehle-Colarusso T, et al: Reporting birth defects surveillance data 1968-2003. Birth Defects Res A Clin Mol Teratol 79: 65-186, 2007.

17. Stecklein SR, Jhingran A, Burzawa J, Ramalingam P, Klopp AH, Eifel PJ and Frumovitz M: Patterns of recurrence and survival in neuroendocrine cervical cancer. Gynecol Oncol 143: 552-557, 2016. 